\title{
Isolated Splenic Metastasis from Rectal Carcinoma: A Rare Occurrence
}

\author{
Sarika Jain $^{a}$ Sumeet Munjal $^{a}$ Rhonda K. Yantiss $^{b}$ \\ Toyooki Sonoda $^{c}$ Thomas J. Fahey ${ }^{c}$ Joseph T. Ruggiero ${ }^{a}$ \\ Alok Anand $^{d}$ Adam Gersten $^{b}$ Stanley J. Goldsmith ${ }^{d}$ \\ Allyson J. Ocean ${ }^{a}$ \\ Departments of ${ }^{\mathrm{a}}$ Medicine, ${ }^{\mathrm{b}}$ Pathology and Laboratory Medicine, 'Surgery, and \\ ${ }^{\mathrm{d}}$ Radiology, Weill Cornell Medical College, New York, N.Y., USA
}

\section{Key Words}

Solitary splenic metastasis $\cdot$ Splenectomy $\cdot$ Chemotherapy $\cdot$ Surveillance

\begin{abstract}
The presence of isolated splenic metastasis in rectal carcinoma is uncommon and usually presents as an asymptomatic mass, noted incidentally on imaging. Splenectomy is usually performed with the goal of curing metastatic disease. It is unclear if adjuvant chemotherapy affords any benefit, and the prognosis is unknown. The case of a young woman is reported, in whom an isolated metastatic lesion in the spleen was discovered 9 months after adjuvant chemotherapy for stage III rectal adenocarcinoma. The patient has remained disease-free for nearly 5 years following splenectomy and chemotherapy. To our knowledge, this is the fourth reported case in the English literature of an isolated splenic metastatic lesion from rectal cancer. We discuss the unique presentation, the importance of post-treatment surveillance, and the implementation of multi-modality treatment strategies in this young patient.
\end{abstract}

\section{Introduction}

In the United States, an estimated 40,000 cases of rectal cancer are expected to occur in 2011. Incidence continues to decrease, as largely evidenced by detection and removal of precancerous lesions through screening. In contrast, in adults younger than 50 years with average risk in whom screening is not recommended, colorectal cancer incidence rates have been increasing by $1.6 \%$ per year since 1998 . Once metastases are detected, the 5-year survival for colorectal cancer approaches a dismal 10\% [1].

Colorectal cancer is unique from other solid tumors in that resection of isolated hepatic, 
peritoneal, or lung metastasis has curable potential in highly selected individuals [2] However, scant literature exists on the incidence and management of isolated splenic metastasis in solid malignancies, including rectal cancer. This case illustrates the importance of post-treatment surveillance, highlights diagnostic and potential multimodality treatment strategies, and discusses the pathogenesis and prognosis of this uncommon presentation in a young woman with rectal cancer.

\section{Case Report}

A 22-year-old female with no significant medical history developed 6 months of hematochezia, which she presumed was secondary to bleeding hemorrhoids. She presented to her physician an additional 2 months after her symptoms progressed to fecal urgency and incomplete evacuation with bowel movements. Digital rectal examination revealed a palpable, nearly circumferential nodular mass, which was tethered to the vaginal apex. Endoscopic examination demonstrated an ulcerated rectal lesion located $10 \mathrm{~cm}$ from the anal verge. Notably polyps were absent. Computed tomography (CT) of the chest, abdomen, and pelvis revealed a lobulated, soft tissue mass in the rectum and perirectal fat stranding without lymphadenopathy or distant metastases. Endoscopic mucosal biopsies demonstrated the presence of a moderately differentiated adenocarcinoma, and concomitant endoscopic ultrasonographic examination suggested that the clinical tumor stage was T4N0. Pertinent laboratory values obtained at the time of the procedure included a carcinoembryonic antigen (CEA) level of $1.1 \mathrm{ng} / \mathrm{ml}$ (normal $<3.0 \mathrm{ng} / \mathrm{ml}$ ). There was no family history of colorectal polyps or carcinoma, and the patient denied smoking or heavy alcohol use. She refused neoadjuvant chemoradiation therapy due to infertility concerns and was treated with neoadjuvant chemotherapy (FOLFOX, 5fluorouracil, leucovorin, and oxaliplatin). This was followed by laparoscopic low anterior resection with total mesorectal excision. Histologic examination of the rectal mass confirmed a moderately differentiated adenocarcinoma, spanning $3.2 \mathrm{~cm}$, with mucinous features (fig. 1). The malignant component infiltrated into the muscularis propria into the perirectal fat, extending to the mesorectal resection margin. Five of seventeen regional lymph nodes contained metastatic carcinoma with focal extracapsular extension (ypT3N2M0). The patient subsequently received adjuvant infusional 5-FUbased chemotherapy.

Nine months after completion of chemotherapy, surveillance imaging with positron emission tomography (PET/CT) imaging revealed an isolated 1-cm hypermetabolic lesion in the spleen with a standardized uptake value (SUV) of 6.4 (fig. $2 \mathrm{a}$ and b), compared to a SUV of 1.7 in the previous year's PET/CT. CEA and complete blood count were normal, and immunophenotyping by flow cytometry of peripheral blood was negative for lymphoproliferative disorder. The patient was otherwise asymptomatic with an ECOG performance status of 0 . She received vaccination against encapsulated bacterial organisms and underwent a laparoscopic-assisted splenectomy. The spleen parenchyma contained a metastatic tumor deposit, histologically similar to the primary rectal carcinoma (fig. 3 ). The patient then received adjuvant chemotherapy (FOLFIRI, 5 -fluorouracil, leucovorin, and irinotecan with bevacizumab) for 6 months. Subsequent surveillance scans have remained negative for recurrent disease at 52 months since completion of adjuvant chemotherapy.

\section{Discussion}

Isolated metastatic involvement of the spleen is rare and sporadic and usually occurs in the setting of concomitant carcinomatosis. Solitary splenic metastases have been reported in lymphoma, melanoma, and gynecologic carcinoma but are a relatively atypical manifestation in rectal cancer [3-6]. In most cases, solitary splenic metastases in rectal cancer are asymptomatic and are detected on imaging or as a result of rising CEA [7]. Berge [3] performed an autopsy study of 7,165 patients and found splenic metastases in only 4.4 and $1.6 \%$ of patients with colonic and rectal carcinoma, respectively; however, the incidence of solitary metastases was not reported. 
To our knowledge, only three cases of rectal carcinoma with isolated splenic metastasis have been reported in the English literature [8]. The first case was that of a 69-year-old man with stage III rectal cancer who developed weight loss, anorexia, and abdominal symptoms related to splenomegaly [6]. The second patient was a 51-yearold female with stage II rectal cancer and solitary splenic metastasis that was detected as a result of increasing CEA levels [9]. The third patient, a 41-year-old male with rectal cancer treated 1 year prior with surgery, had the most catastrophic presentation, suffering a splenic rupture due to a massively enlarged spleen. Histology revealed diffuse involvement of the spleen with mucin-producing adenocarcinoma, and no other distant organs were involved [10]. These patients were treated with splenectomy, and subsequent chemotherapy was not administered. In our case, the young woman was asymptomatic and her CEA remained low at progression; the splenic lesion was identified on a surveillance PET/CT. We acknowledge that CT is the standard method for assessing recurrence, and the lesion may also have been seen on a CT with intravenous contrast.

The spleen as a site of metastases in rectal cancer is generally uncommon, presumably as a result of anatomic, functional, and immunologic factors. Carcinomas may spread to the spleen via three possible routes: hematogenous seeding from the splenic artery or vein, lymphovascular dissemination, and direct extension from the peritoneum. Vascular dissemination is least common, owing to the sharply angulated nature of the splenic and celiac arteries, the rhythmic contraction of the spleen, and a lack of afferent lymphatic vessels in the splenic parenchyma [11, 12]. Furthermore, the spleen is considered a hostile soil for neoplastic implantation, perhaps related to the immunologic surveillance function of the spleen [13]. The humoral substances and immunologically competent cells within the spleen have been hypothesized to destroy tumor cells as they reach the organ and decrease adherence of tumor cells to the basement membrane, respectively [14].

Management of this unique entity is not well established given the small amount of case reports available. The preferred surgical management appears to be splenectomy, supported by a survival benefit seen with metastasectomy in other cancers [7].

Moreover, post-metastasectomy chemotherapy has been shown to decrease recurrences and prolong survival in colorectal cancer patients with distant metastases [15]. Extrapolating from these data, administration of post-splenectomy chemotherapy can be a consideration in fit patients. Prognosis in rectal cancer patients with isolated solitary splenic metastasis is unknown, but the mean reported survival interval in a recent meta-analysis of 26 colorectal cancer patients with isolated splenic metastasis reached 19.5 months [7].

\section{Conclusion}

This is a case of an isolated, solitary splenic metastasis from rectal carcinoma, a rare entity. Diagnosis can prove to be difficult as the majority of patients are asymptomatic, underscoring the importance of surveillance with both imaging and CEA levels. Management strategies are not conclusive and long-term prognosis is unknown. Our patient has been disease-free for nearly 5 years after splenectomy, and though it is 
unknown if post-splenectomy systemic chemotherapy has increased her overall survival, it could be considered in young patients with a good performance status.

\section{Disclosure Statement}

None of the authors have any conflicts of interest to disclose.

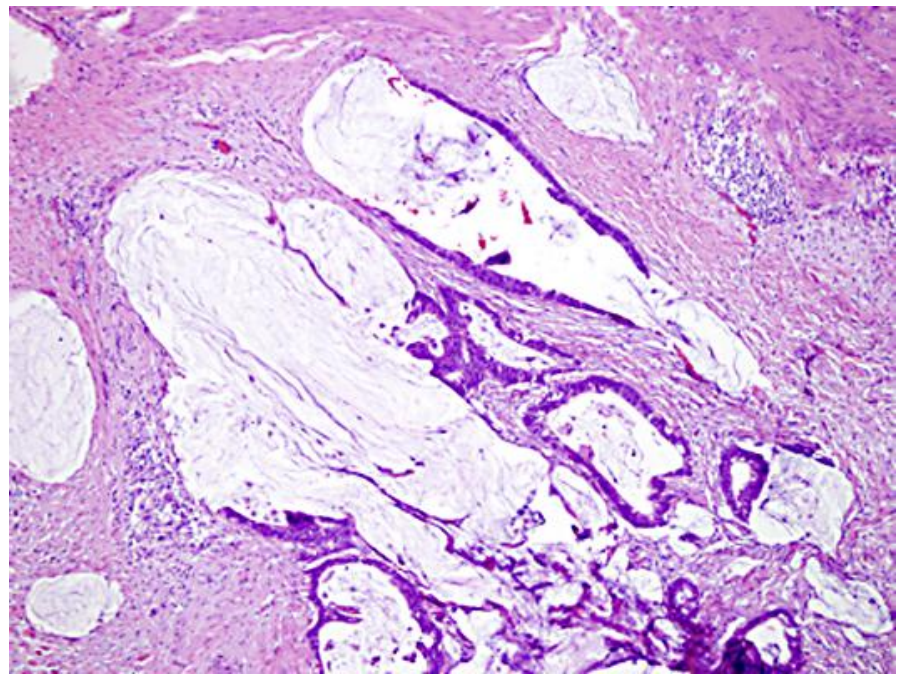

Fig. 1. The resected rectal cancer contained moderately differentiated malignant glands, many of which were distended with abundant mucin as seen at $100 \mathrm{HPF}$. Free pools of dissecting mucin were present within the tumor as well but accounted for less than $10 \%$ of the total tumor volume. 


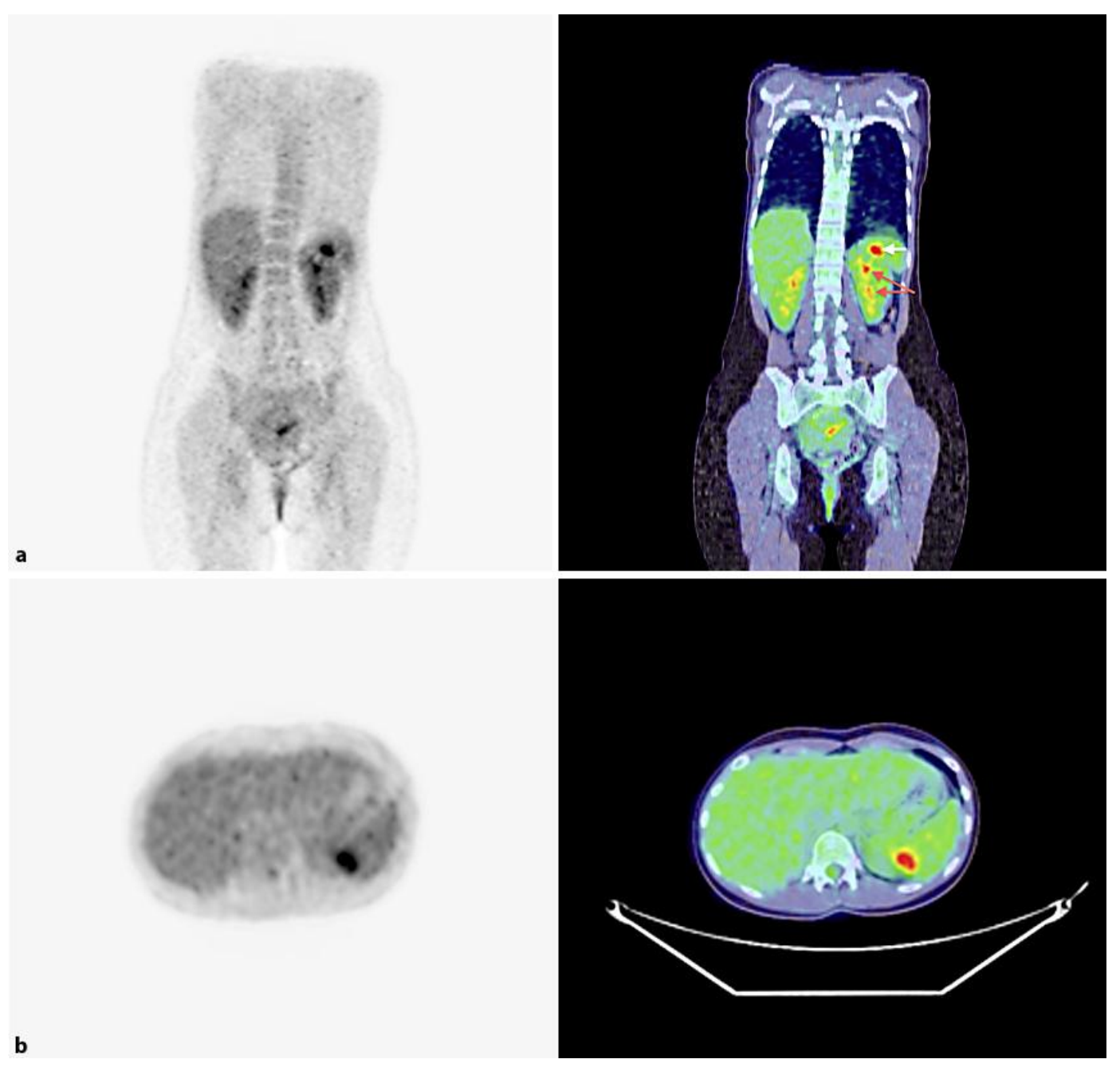

Fig. 2. a Coronal F-18 fluorodeoxyglucose (FDG) PET (left) and fused PET/CT (right) images demonstrate a hypermetabolic focus in the spleen, which corresponds to a low attenuation lesion on $\mathrm{CT}$. The single white arrow indicates the splenic lesion. Pink arrows indicate physiologic urinary excretion of radiotracer within the renal calyces. $\mathbf{b}$ Transaxial F-18 FDG PET (left) and fused PET/CT (right) images at the level of the upper pole of the left kidney demonstrate FDG-avid mass within the posterior medial aspect of the midsection of the spleen. 


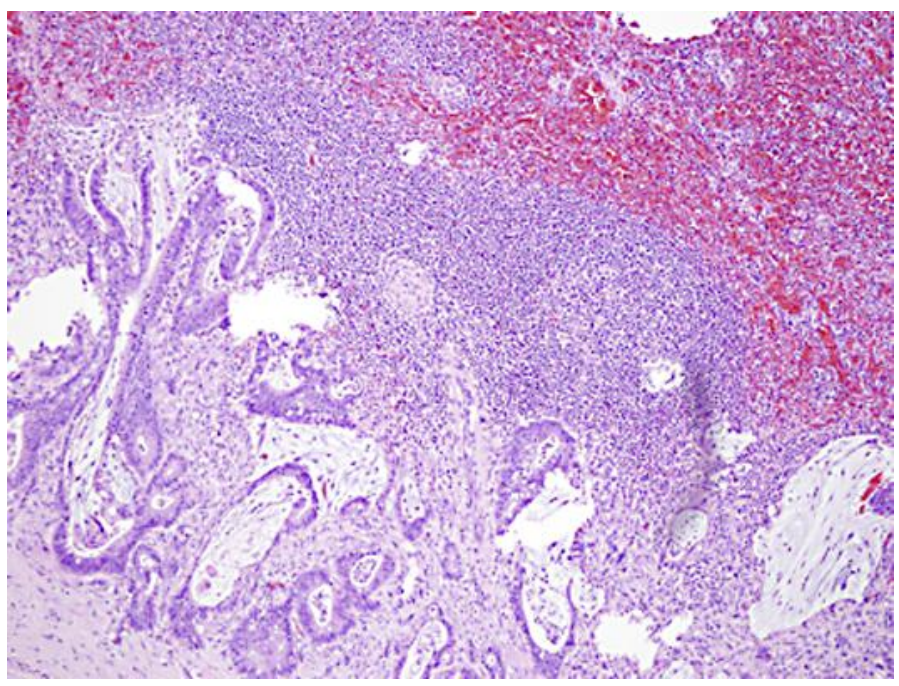

Fig. 3. The spleen contained a metastatic tumor deposit within the parenchyma, unassociated with the overlying capsule. The tumor was histologically similar to the primary rectal carcinoma.

\section{References}

1 American Cancer Society: Cancer Facts and Figures 2011. Available at http://www.cancer.org/acs/groups/content/@epidemiologysurveilance/documents/document/acspc029771.pdf (accessed June 22, 2011).

-2 Kuo LJ, Leu SY, Liu MC, et al: How aggressive should we be in patients with stage IV colorectal cancer? Dis Colon Rectum 2003;46:1646-1652.

-3 Berge T: Splenic metastases. Frequencies and patterns. Acta Pathol Microbiol Scand A 1974;82:499-506.

4 Shocket E, Dembrow VD: Splenic metastases from a melanoma of the nasal mucosa. The only thoracoabdominal manifestation. Am J Surg 1963;106:949-953.

$\rightarrow 5$ Waller RM 3rd, Fajman WA: An unusual cause of an isolated, focal splenic defect demonstrated by liverspleen scintigraphy. Clin Nucl Med 1982;7:5-7.

-6 Dunbar WH, Beahrs OH, Morlock CG: Solitary splenic metastasis incidental to rectal carcinoma: report of a case. Mayo Clin Proc 1969;44:40-45.

7 Abi Saad GS, Hussein M, El-Saghir NS, et al: Isolated splenic metastasis from colorectal cancer. Int J Clin Oncol 2011;16:306-313.

-8 Pisanu A, Ravarino A, Nieddu R, et al: Synchronous isolated splenic metastasis from colon carcinoma and concomitant splenic abscess: a case report and review of the literature. World J Gastroenterol 2007;13:5516-5520.

9 Capizzi PJ, Allen KB, Amerson JR, et al: Isolated splenic metastasis from rectal carcinoma. South Med J 1992;85:1003-1005.

10 Achuthan R, Joseph A, Haray PN: Splenic metastasis from a rectal tumour: an unusual presentation. Ann R Coll Surg Engl 1999;81:139.

11 Cavallaro A, Modugno P, Specchia M, et al: Isolated splenic metastasis from colon cancer. J Exp Clin Cancer Res 2004;23:143-146.

12 Harman JW, Dacorso P: Spread of carcinoma to the spleen, its relation to generalized carcinomatous spread. Arch Pathol (Chic) 1948;45:179-186.

13 Indudhara R, Vogt D, Levin HS, et al: Isolated splenic metastases from colon cancer. South Med J 1997;90:633-636.

14 Nicolson GL: Cancer metastasis: tumor cell and host organ properties important in metastasis to specific secondary sites. Biochim Biophys Acta 1988;948:175-224.

15 Nozawa H, Kitayama J, Sunami E, et al: FOLFOX as adjuvant chemotherapy after curative resection of distant metastases in patients with colorectal cancer. Oncology 2011;80:84-91. 